\title{
van der Waals
}

Johannes Diderik van der Waals was born on 23 November 1837 at Leiden, Netherlands, to Jacobus van der Waals and Elisabeth née van den Burg [1]. He was the eldest of ten children (four daughters and six sons). It is interesting to note that the youngest of the six brothers, Henrik Gerardus van der Waals was a translator of Sanskrit. Jacobus was a carpenter and his family's scanty means implied that Johannes had only primary school education. It appears that there was no public secondary education in Netherlands or in other European countries in those days. University education was beyond reach for van der Waals as he had to work for earning his daily bread until he was about 25 years old. Besides, he had not learnt the classical languages, which was a prerequisite for entering a University those days. van der Waals worked as a school teacher following his primary education. Starting from late 18th century, many independent public associations played an important role in Dutch public education. The Leiden community supported the founding of a 3-year industrial evening school for working class youth and this helped van der Waals continue his studies. Though he was not allowed to take academic examinations at the University yet, van der Waals continued studying at Leiden University in his spare time from 1862, when he was already 25. This continued till 1865 and during this period he obtained teaching certificates in physics and mathematics. He worked as a teacher at Deventer in 1864 and moved to The Hague in 1866 [2]. A carpenter's son had become a physics teacher who continued his journey to become an important physicist of all time.

A new legislation, allowing science students enrolled in the University to be exempted from the classical language requirement, helped van der Waals to sit for University examinations. He was 36 when he finally obtained his $\mathrm{PhD}$ in 1873 [1] (this would be considered too old in today's academic institutes in India!). The title of his thesis was Over de Continuïteit van den Gas-en Vloeistoftoestand (On the continuity of the gas and liquid state). It is not surprising that van der Waals' thesis has become a classic in physics literature though he lacked expertise in classical languages. In his thesis, van der Waals introduced the equation of state which has made him immortal. There is no student of science today graduating in any discipline of science anywhere in the world without learning the 'van der Waals equation'. The importance of this equation can be understood by reading the comment made by none other than James Clerk Maxwell in Nature (as quoted in [2]): "that there can be no doubt that the name of van der Waals will soon be among the foremost in molecular science". Today 'molecular science' is a term used to describe chemistry [3] and van der Waals forces are indeed very important in chemistry. However, the real impact of his equation can be seen by the title of a recent book by Adrian Parsegian: Van der Waals Forces: A Handbookfor Biologists, Chemists, Engineers and Physicists [4]. He was 
awarded the Nobel Prize in Physics for the year 1910 "for his work on the equation of state for gases and liquids". This year marks the centenary of this award. How did van der Waals arrive at this equation?

According to van der Waals himself, it was the treatise by Clausius On the nature of the motion which we call heat (1857) that led him to the equation. The book by Kipnis et al. [1] quotes van der Waals' close associate Ph A Khonstamm: "we can be sure that by 1865 van der Waals had been captivated by the conclusions of Clausius and was familiar with the new ideas of molecular theory". Those days, however, molecules were assumed to be point masses occupying no space and hence the suggestion that 'the temperature is nothing other than the energy of their (molecule's) movement' met with a critical, but wrong, objection by C H D Buys Ballot [1]. He pointed out that the rate of mixing of gases by diffusion was much slower than what was consistent with the high speeds and rectilinear paths required by the kinetic theory. Clausius quickly abandoned his original postulate that gas molecules are of infinitesimal size and postulated instead that the gas molecules have large enough diameters and cannot move very far without hitting another one. This led to the fundamental concept of a mean free path of gas molecules. Clausius had indeed pointed out that intermolecular attractions that keep the molecules together in liquid were the same as those operating in between molecules in the gas phase, but only dominant when the pressure is high. Clausius had used this idea to differentiate the gaseous state from the liquid and solid states of matter. The genius in van der Waals realized that the same idea should lead to 'continuity of the liquid and gas state' thereby identifying the unifying forces. Thus he modified the ideal gas law, which assumes that the molecules are infinitesimally small and have no forces acting between them, leading to the well-known relation $P V=n R T$. Here, $P$ is the pressure, $V$ is the volume of the container, $n$ is the number of moles of gases present in the container, $R$ is the universal gas constant and $T$ is the temperature. van der Waals pointed out that the actual pressure felt by the container walls would be less if the molecules hitting the walls are pulled back due to the attraction from the other molecules. He also pointed out that the actual volume available for the gas molecules would be less as the molecules have finite size and they occupy some volume. That is, for the ideal gas law to be valid $P$ should be increased and $V$ should be decreased. He introduced two constants $a$ and $b$ to make this correction and proposed the equation named after him:

$$
\left(P+\frac{n^{2} a}{V^{2}}\right)(V-n b)=n R T .
$$

The volume occupied by the gas molecules is directly proportional to the number of moles and the pressure depends on the square of $n / V$, the number of moles per unit volume, leading to the corrective terms as given by van der Waals. 
That van der Waals was convinced of the existence of finite size molecules echoed in his Nobel address: [1] "It will be perfectly clear that in all my studies I was quite convinced of the real existence of molecules, that I never regarded them as a fragment of imagination, nor even as mere centres of force effects. I considered them to be the actual bodies". van der Waals equation not only leads to fundamental understanding of the states of matter, it has had practical consequences. In the Award Ceremony on December 10, 1910, Prof. O Montelius, President of the Royal Swedish Academy of Sciences, said the following: "Modern refrigeration engineering, which is nowadays such a potent factor in our economy and industry, bases its vital methods mainly on Van der Waals' theoretical studies".

van der Waals also derived the law of corresponding states in 1880 which is considered his second great discovery. This led to a general form of an equation of state applicable for all substances based on their critical parameters. This law guided the experiments that ultimately led to the liquefaction of hydrogen by J Dewar in 1898 and helium (the last gas to be liquefied) by H Kamerlingh Onnes in 1908. The latter was a student of van der Waals who went on to win the Nobel Prize in Physics in the year 1913. In 1890, van der Waals published a treatise on the theory of binary solutions, which combined his equation of state with the second law of thermodynamics.

The constant $a$ was introduced by van der Waals to account for the fact that there are attractive forces between molecules. Consequently all intermolecular attractive forces were called van der Waals forces. The nature of these forces was not understood in the 19th century and van der Waals did not mention anything about them. The other constant $b$ naturally leads to van der Waals volume for atoms/molecules. When gases condense forming liquid and then solid states, the molecules are closely packed. Analysis of the crystal structure can lead to accurate estimation of the distances between atoms from neighbouring molecules that are not chemically bound. Pauling analyzed a large number of such structures and introduced 'van der Waals radius' for most atoms [5]. It defines a minimum radius for each type of atom such that other non-bonded atoms in the vicinity do not get any closer to the atom in concern. After Pauling, Bondi derived van der Waals radius for many atoms using several methods [6]. Thus van der Waals not only has the equation he derived named after him, but also a volume and radius for atoms/molecules derived by other eminent scientists named after him. The author of this article is also writing a series of articles on van der Waals molecules (atoms/molecules bound by van der Waals forces), not known during van der Waals' time, in Resonance, two of which have appeared earlier [3,7] and the third one appears in this issue on p.667. This may be a unique feature about the legendary van der Waals, who was born to a carpenter and had to wait till he was 36 to obtain his $\mathrm{PhD}$ due to the prevailing social conditions. van der Waals died in 
Amsterdam on March 8, 1923. However, he lives to this day and will live forever in his equation of state.

\section{Suggested Reading}

Most of the historical details mentioned in this article are based on [1,2 and 4].

[1] A Ya Kipnis, B E Yavelov, and J S Rowlinson, Van der Waals and Molecular Science, Oxford University Press, Oxford, 1996.

[2] J D van der Waals - Biography, http://nobelprize.org/nobel_prizes/physics/laureates/1910/waals-bio.html

[3] E Arunan, Resonance, Vol.14, No.4, p.346, 2009.

[4] V Adrian Parsegian, Van der Waals Forces: A Handbook for Biologists, Chemists, Engineers and Physicists, Cambridge University Press, Cambridge, 2006.

[5] L Pauling, The Nature of the Chemical Bond, Cornell University Press, Ithaca, 1960.

[6] A Bondi, J. Phys. Chem., Vol.68, p.441, 1964.

[7] E Arunan, Resonance, Vol.14, No.12, p.1210, 2009.

E Arunan

Department of Inorganic and Physical Chemistry Indian Institute of Science, Bangalore 560 012, India.

Email:earunan@gmail.com

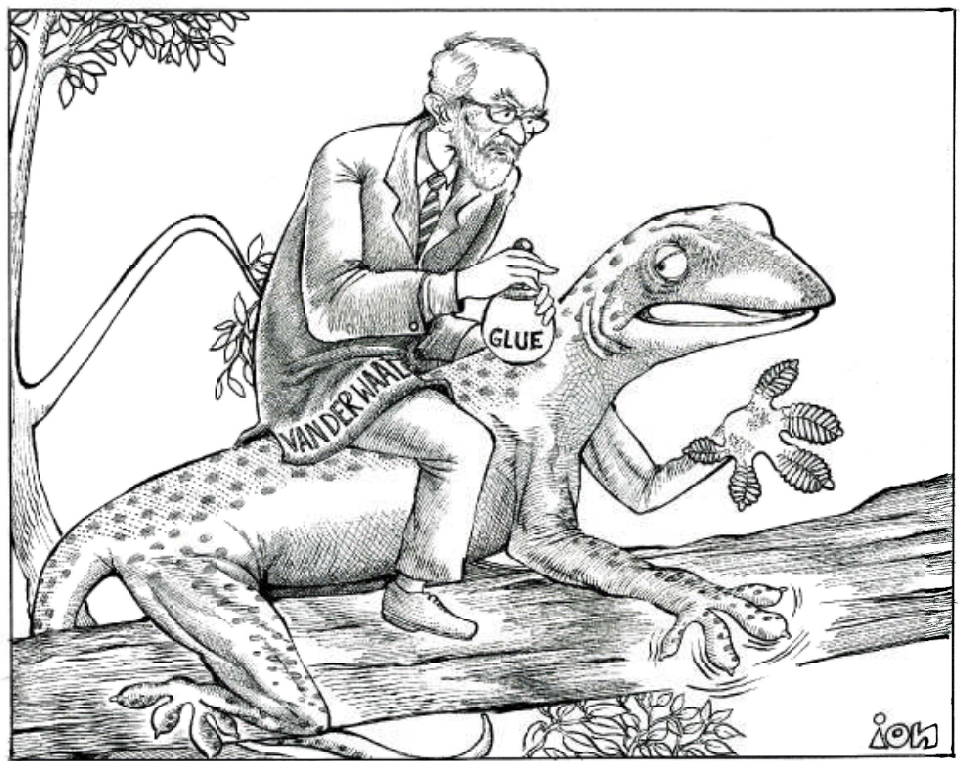

Have confidence, Prof. van der Waals, I will never fall - the hairs in my feet stick to the surface using your forces, and is better than any glue that man has ever made. 